\title{
Effects of distance between standard and comparison lines on the Müller-Lyer illusion
}

\author{
A. W. PRESSEY and V. Di LOLLO \\ Uriversity of Manitoba, Winnipeg, R3T.2N2, Canada
}

\begin{abstract}
The Müller-Lyer illusion was measured as a joint function of gap between fins and shaft and distance between standard and comparison lines. The results showed that the traditional function relating illusion to gap was present, but that the shape of the function was greatly influenced by the distance between standard and comparison lines. The mathematical formulation of assimilation theory provided adequate predictions under the assumption that the attentive field changes as a constant ratio of target size.
\end{abstract}

One of the most popular targets for investigating visual distortions is the Müller-Lyer configuration in which the inward pointing fins (arrowheads) or outward pointing fins (arrow feathers) are drawn at the ends of a standard line. The arrowheads produce phenomenal shrinkage and the arrow feathers produce phenomenal expansion of the standard line.

The effects of a large number of stimulus variables on the Müller-Lyer illusion have been investigated. These variables include length, angle, color, and number of fins; temporal and spatial separation between shaft and fins and target size (Pressey \& Wilson, 1977; Robinson, 1972). However, one stimulus variable that has not been investigated is the location of the comparison line in relation to the standard line. It would be expected that the location of the comparison line would have a substantial effect on the illusion for two reasons. First, it has been shown that the Ponzo illusion is altered when the position of the comparison line is changed (Pressey, 1974). The second reason is a theoretical one. The assimilation theory of geometric illusions (e.g., Pressey, Butchard, \& Scrivner, 1971; Pressey \& Murray, 1976) contends that spatial deployment of attention (which affects the amount of illusion) is partly determined by the positions of the standard and comparison lines. Thus, any systematic change in the relationship between the two lines should result in a systematic change in illusion.

The major aim of this investigation was to determine the effects of varying the distance between the standard and comparison lines on reversed forms of the arrowhead Müller-Lyer illusion. The reversed illusion is produced by imposing gaps of various sizes between the shaft and the fins (Fellows, 1967; Yanagisawa, 1939). These targets were chosen because data relating magnitude of illusion to size

This study was supported by the National Research Council of Canada (A0-177). We wish to express our appreciation to Alexander. Wilson for his aid in this study. of gap were already available in our laboratory (Pressey \& Bross, 1973; Pressey, Di Lollo, \& Tait, 1977). A second reason for choosing these targets was that the quantitative formulation of assimilation theory predicts patterns of results on the reversed Müller-Lyer effect quite well (Pressey \& Murray, 1976; Pressey, Di Lollo, \& Tait, 1977). Thus, a second objective of this study was to determine what variations in the quantitative formulation of the theory would be necessary to provide a fit for whatever changes occurred as distance between standard and comparison line was varied.

\section{METHOD}

\section{Subjects}

The subjects were 56 experimentally naive undergraduate students who provided their services as part of a class experiment in a laboratory course. None had any obvious visual impairment or grossly defective vision.

\footnotetext{
Apparatus

All visual displays were generated by a PDP-8/A computer, which also performed all response acquisition and scoring functions. The displays were first plotted on a Tektronix 602 point plotter and were then distributed, through a video distribution system, to eight separate cubicles. This enabled eight subjects to be tested separately and simultaneously. Each cubicle contained a Sony video monitor mounted on a shelf at about eye level of a sitting observer. A chinrest was mounted on a table directly in front of the monitor so that the distance of the display surface from the subject's eyes was about $128 \mathrm{~cm}$.

Each display consisted of two portions: an ingoing MüllerLyer test figure, centered in the upper portion of the display surface, and a comparison line located in the lower portion. The test figure consisted of a horizontal shaft, $75 \mathrm{~mm}$ long and and bounded by two fins pointing away from the center of the figure. The internal angle of each fin was $53^{\circ}$, and the length of each component segment was $19 \mathrm{~mm}$. The comparison line, whose initial length varied randomly on each trial between 56 and $94 \mathrm{~mm}$, extended to the right of an original point which varied randomly within a stretch of $15 \mathrm{~mm}$ to either side of an imaginary point below the leftmost point of the shaft. The degree of vertical separation between shaft and comparison lines varied with the experimental condition, as explained below. The thickness of all lines was approximately $1 \mathrm{~mm}$. All viewing was done in semidarkness. A set of seven response buttons, only three of
} 
which were used in this experiment, was placed on the table within reach of the subject. Two response buttons allowed the subject to increase or decrease, respectively, the length of the comparison line in discrete steps of $.586 \mathrm{~mm}$. Depression of the third response button terminated a trial and initialized conditions for the next trial.

\section{Procedure}

Two aspects of the display were varied systematically: the width of gap between ends of shaft and apices of fins in the MüllerLyer figure, and the vertical separation between shaft line and comparison line. The former factor was varied within subjects, the latter between subjects. Size of gap was varied between zero and $33.75 \mathrm{~mm}$ in steps of $3.75 \mathrm{~mm}$; in addition, there was a control condition in which the fins were not displayed. This yielded a total of 11 within-subject conditions.

Vertical separation between shaft and comparison lines varied systematically across the eight cubicles from $39.4 \mathrm{~mm}$ in Cubicle 1 to $124.8 \mathrm{~mm}$ in Cubicle 8 , in successive steps of $12.2 \mathrm{~mm}$ for each intervening cubicle. The distance between shaft and comparison lines were thus $39.4,51.6,63.8,76.0,88.2,100.4,112.6$, and $124.8 \mathrm{~mm}$, for the displays in Cubicles 1 to 8 , respectively. In practice, this was done by plotting the Müller-Lyer figure and all eight comparison lines in every cubicle. However, each display surface was covered with a matt black cardboard cover having two horizontal openings which allowed unimpaired vision of the Müller-Lyer figure and of only one of the eight comparison lines. The response buttons in any given cublcle allowed control of the length of the comparison line that was visible in that cubicle.

Eight subjects, chosen randomly from the pool of 56 subjects, were tested simultaneously, one in each cubicle. Data were thus collected simultaneously on each of the eight between-subject conditions defined by the eight levels of vertical separation between shaft and comparison line. This procedure was repeated with a new set of eight subjects for a total of seven experimental sessions. Each experimental session comprised 55 trials consisting of five presentations of the display at each of the 10 gap sizes and 5 control trials without the fins. The 55 trials occurred in a different random order in each of the seven sessions and were completed in about $20 \mathrm{~min}$. On each trial, the subject's task was to adjust the length of the comparison line to match that of the shaft of the Muller-Lyer figure. Upon termination of a trial, the program scored the responses by subtracting the length of the shaft from the length of the comparison line as set by each subject, separately. A negative score thus indicated underestimation and a positive score overestimation of the length of the shaft.

\section{RESULTS AND DISCUSSION}

For each subject, an illusion score was calculated by averaging the five responses at each condition and then subtracting the average response to the control line.

The magnitude of illusion, as a joint function of gap between shaft and fins and of distance between standard and comparison lines is shown in Figure 1. For clarity of illustration, results from adjacent comparison lines are averaged. Analysis of variance indicated that the variable of gap was significant $[F(9,936)=271.2, p<.01]$; the position of comparison line was significant $[F(7,104)=2.54$, $\mathrm{p}<.02$ ], and the interaction between gap and position of comparison line was significant $[F(63,936)=3.22, p<.01]$. Thus, the results reveal

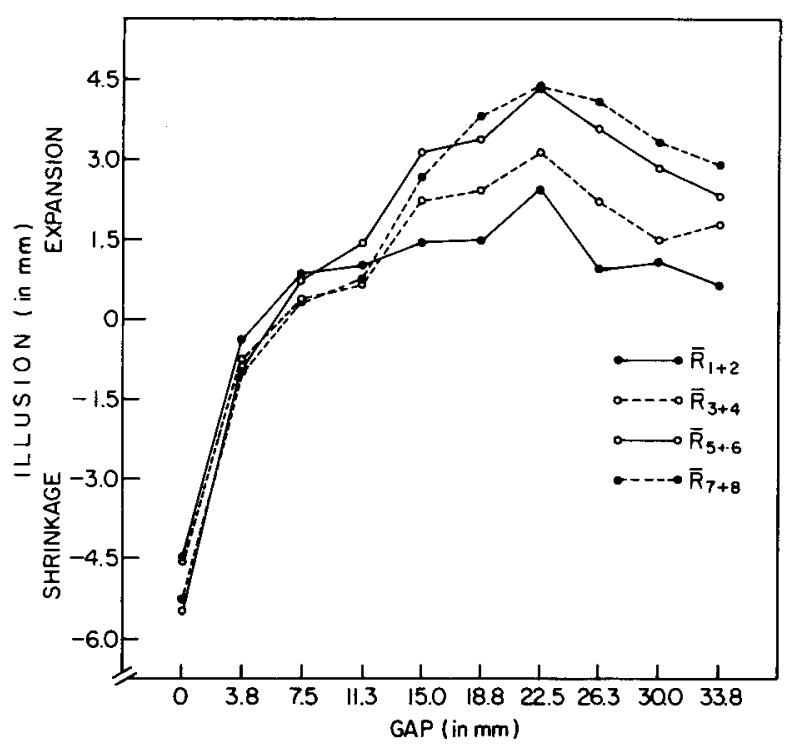

Figure 1. Müller-Lyer illusion as a joint function of gap between fins and shaft and distance between standard and comparison lines (R). Adjacent distances are averaged for clarity of presentation.

quite strikingly that the distance between comparison line and the standard line has a robust effect on the magnitude of illusion.

A survey of the theories of illusion shows that, with the possible exception of Piaget's (1969) and Pressey's (1972) theories, none is capable of explaining the data obtained in this experiment. The main reason, of course, is that most theories ignore the role of attention.

Piaget has argued that attention plays a substantial role in illusions and endorsed the following two generalizations. Ths first is that "an element is overestimated to the extent that it is seen distinctly and underestimated to the extent that it lacks distinctness" and the second is that "an element to which attention is paid is thereby overestimated" (Piaget, 1969; pp. 76-77). However, it is difficult to see how either of these generalizations could explain the nonmonotonic results shown in Figure 1. Indeed, the problem is amplified when one considers the fact that the data in Figure 1 are evaluated against control lines which themselves should have displayed the effects that Piaget postulated.

Pressey's assimilation theory includes an attentive field postulate which states that the effectiveness of a contextual stimulus in distorting a standard stimulus decreases as the contextual stimulus moves from the center to the periphery of the field. The center of the field is operationally defined as the midpoint between the two most spatially disparate points of the standard and comparison magnitudes and the entire field is assumed to be circular. A schematic representation of an attentive field is 
shown in Figure $2 \mathrm{~A}$. The distance, $\mathrm{a}$, is the radius of the "minimum attentive field" (i.e., the minimum size that is logically necessary to postulate if the subject is to perform the task), and b is the optimum attentive field, i.e., the size that is necessary to provide the best fit to empirical data.

The main effect of gap shown in Figure 1 can be readily explained by referring to Figure $2 \mathrm{~A}$. When there is no gap, an illusion of shrinkage will occur because the standard line assimilates to (or is averaged with) the shorter magnitudes that the fins define (Pressey, 1971). However, as gap increases, the contextual magnitudes produced by the fins will eventually become longer than the standard line, and the illusion should reverse and become an illusion of expansion which should continue to increase (Pressey, 1972). However, as gap increases, these contextual magnitudes have a greater and greater probability of not being utilized, because they fall outside the attentive field. With very large gaps, then, the expansion effect should begin to decline, and it does, as can be seen in Figure 1.

Consider now the target shown in Figure 2B. In this case, the only stimulus change is that the distance between standard and comparison lines has increased. One consequence is that the minimum attentive field (c) has increased. Because $c$ has increased, it seems reasonable to suppose that $d$ will also increase. If we make the simple assumption that the optimum field is a constant ratio of the minimum field $(a / b=c / d=k)$, then the situation shown in Figure 2B will obtain. But now one fact stands out very clearly. With the same size gap, the contextual magnitudes are closer to the center of the attentive field in Figure $2 \mathrm{~B}$ than they are in Figure 2A. Therefore, with large gaps, there will be a larger expansion illusion in Figure 2B than in Figure 2A. Thus, on the assumption that attentive field increases, as a constant ratio of the minimum field, the data in Figure 1 seem predictable.

In order to provide a more rigid test of assimilation theory, an attempt was made to predict the patterns of results shown in Figure 1 with the mathematical formula that has been developed for the assimilation theory (Pressey \& Murray, 1976). The formula is as follows:

$$
I=\frac{1}{N_{j=1}} \sum_{j=1}^{N}\left(1-\frac{D_{c j}}{D_{p}}\right)\left(1-\frac{D_{c j}^{\prime}}{D_{p}^{\prime}}\right)\left(L_{c j}-L_{s}\right),
$$

where $L_{c j}$ is the length of contextual magnitude $j$, corresponding to the $\mathrm{j}$ th imaginary line, parallel to the shaft, bounded by corresponding $j$ points on the opposite fins of the figure; $L_{s}$ is the length of the standard magnitude (i.e., shaft length); $D_{c j}$ is the distance from the center of the attentive field to the most distant point of contextual magnitude $j ; D_{p}$ is the distance from the center to the periphery of the attentive field; $D_{c j}^{\prime}$ is the distance from the center of the interactive field to the contextual magnitude $\mathrm{j} ; \mathrm{D}_{\mathrm{p}}^{\prime}$ is the distance from the center to the periphery of the interactive field; and $N$ is the total number of contextual magnitudes sampled by this estimation procedure, all of which fall entirely within both the attentive and the interactive fields.

In order to understand the formula, it is helpful to refer to Figure $2 \mathrm{C}$.

The center of the attentive field was operationally defined as the intersect of the diagonals connecting the opposing end points of the standard line and the comparison line. In Figure $2 \mathrm{C}$, the center of the attentive field is marked with a dot. The center of the interactive field was operationally and arbitrarily defined as one tip of the standard line (marked $\mathrm{X}$ in Figure $2 C$ ). Thus, any one $D_{c j}^{\prime}$ was the distance from the tip of the standard line to any $j$ point on the proximal fin. In Figure $2 \mathrm{C}$, it is the distance
A

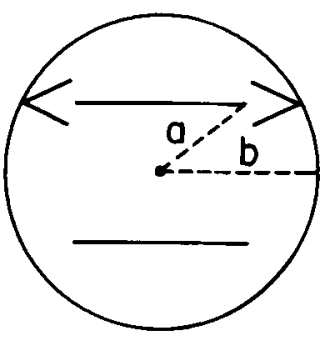

$B$

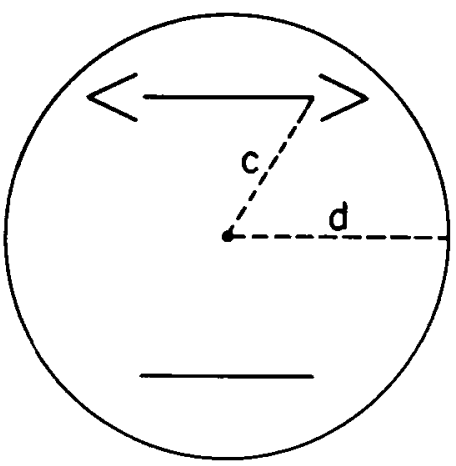

C

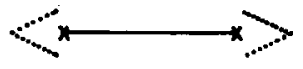

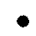

Figure 2. (A) A reversed ingoing Müller-Lyer target with a hypothetical attentive field of radius, b. (B) A reversed ingoing Müller-Lyer illusion in which distance between standard and comparison lines is increased. (C) Schematic representation of target used to predict illusion scores. 
from a cross to one of the dots forming the proximal fin. The rationale for the operational definitions of attentive and interactive fields has been provided by Pressey (1971) and Pressey and Murray (1976).

The values of the stimulus variables were derived from the targets that were employed in this study, and the values for the organismic variable of interactive fields were those that were previously found to be successful for predicting the parallel lines, the Ponzo, and the reversed Müller-Lyer illusions (Pressey \& Murray, 1976). Thus, the standard magnitude was $75 \mathrm{~mm}$ and the values of the contextual magnitudes were determined by sampling points every $0.1 \mathrm{~mm}$ along the oblique lines and then joining the contralateral points. This gave a series of lines which varied in length and were parallel to the standard line.

For each of the distances between standard and comparison line, four values of the interactive field $\left(D_{p}^{\prime}\right)$, ranging from 10 to $40 \mathrm{~mm}$ in steps of $10 \mathrm{~mm}$, were employed. Also five values of the attentive field $\left(D_{p}\right)$ were employed, but these values varied as a constant ratio of the minimum attentive field. The ratios were based on the ratios that had been used previously to predict illusion data (Pressey, Di Lollo, \& Tait, 1977; Pressey \& Murray, 1976). Thus the prediction of each point was the average of a matrix of 20 scores. It is important to emphasize that, in predicting the effect of gap on illusion, the values of the attentive and interactive fields were constant across matrices; but, in predicting the effect of distance between standard and comparison lines, the values of the interactive fields remained constant while the value of attentive fields changed as a constant ratio of the minimum attentive field.

The predicted data are shown in Figure 3. It is clear that the patterns of results are quite similar to those shown in Figure 1. The interaction between gap and distance between standard and comparison lines is particularly well predicted. In addition, it is interesting to note that the predicted curves all peak at the same gap size, which is similar to what occurs in the obtained data ( although the gap at which the peak occurs is slightly different in the two sets of data). At any rate, the fit between the obtained and predicted results provides evidence for the role of spatial deployment of attention in visual perception. In addition, it appears that the degree of deployment is systematically related to stimulus variables such as target size. Indeed, this experiment may provide the basis for a major extension of assimilation theory. One difficulty of the theory has been its inability to predict what would happen if, for example, the length of the standard was varied. And the major drawback to such a prediction was

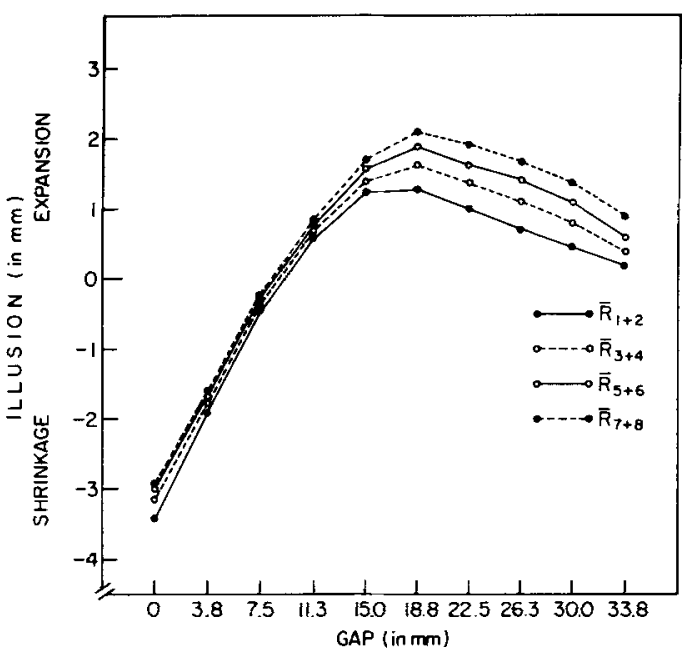

Figure 3. Predicted Müller-Lyer illusion as a joint function of gap between fins and shaft and distance between standard and comparison lines (R). Adjacent distances are averaged to conform to the presentation of results in Figure 1.

an inability to specify, in an a priori manner, what sizes of attentive fields should be employed. The present study indicates that the optimum attentive field may be a constant ratio of the minimim attentive field. The question, however, is an empirical one and it may be useful to attempt to verify the generalization more directly.

It should also be noted that, in predicting the results of this experiment, the sizes of the interactive fields were not varied. (The interactive field is a construct which specifies the manner in which the elements interact as a function of the spatial distance between those elements. It is argued that the effectiveness of a contextual feature in distorting a standard feature decreases as the spatial distance between the two features increases.) The results seem to indicate that increasing the distance between the standard and comparison lines does not affect the interactive field. In other words, the size of the interactive field specifies the ability of an observer to gate out a contextual feature when it is close in space to a standard feature, and this ability is not altered by changing the distance between the standard and comparison lines. This is, of course, consistent with a "common sense" conception of an interactive field. But what is more important is that the technique of increasing distance between standard and comparison lines may provide a way of uniquely changing attentive fields without confounding other variables. If this were the case, then a technique of manipulating the degree to which individuals deploy attention would be available. Such a technique would be especially useful in analyzing the processes involved in perceptual 
learning. One of the most stable facts about visual illusions is that the amount of distortion changes with repeated trials. One interpretation of this effect is that it is due to the restriction of attention (Lewis, 1908; Newbigging, 1965). If this were the case, then transfer of training tasks (in which tasks vary in distance between standard and comparison lines) could be employed to induce large or small attentive fields, and hence large or small illusions.

\section{REFERENCES}

Fellows, B. J. Reversal of the Muller-Lyer illusion with changes in the length of the inter-fins line. Quarterly Journal of Experimental Psychology, 1967, 19, 208-214.

LEwIS, E. O. The effect of practice on the perception of the MullerLyer illusion. British Journal of Psychology, 1908, 2, 294-306.

Newbigging, P. L. Attention and perceptual learning. Canadian Psychologist, 1965, 6, 309-331.

Piaget, J. The mechanisms of perception. New York: Basic Books, 1969.

Pressey, A. W. An extension of assimilation theory to illusions of size, area, and direction. Perception \& Psychophysics, $1971,9,172-176$
Pressey, A. W. The assimilation theory of geometric illusions: An additional postulate. Perception \& Psychophysics, 1972. 11, 28-30.

Pressey, A. W. Evidence for the role of attentive fields in the perception of illusions. Quarterly Journal of Experimental Psychology, 1974, 26, 464-471.

Pressey, A. W., \& Bross, M. Assimilation theory and the reversed Müller-Lyer illusion. Perception, 1973, 2, 211-217.

Pressey, A. W., Butchard, N., \& Scrivner, L. Assimilation theory and the Ponzo illusion: Quantitative predictions. Canadian Journal of Psychology, 1971, 25, 486-497.

Pressey, A. W., \& Murray, R. Further developments in the assimilation theory of geometric illusions: The adjacency principle. Perception \& Psychophysics, 1976, 6, 536-544.

Pressey, A. W., Di Lollo, V., \& Tait, R. W. Effects of gap size between shaft and fins and of angle of fins on the Müller-Lyer illusion. Perception, 1977, 6, 435-439.

Pressey, A. W., \& Wilson, A. E. The hydra-headed MuellerLyer effect: A theoretical puzzle. Perception \& Psychophysics, $1977,22,392-394$.

Robinson, J. O. The psychology of visual illusions. London: Hutchinson, 1972.

YANAGISAWA, N. Reversed illusion in the Müller-Lyer illusion. Japanese Joumal of Psychology, 1939, 14, 321-326.

(Received for publication May 15, 1978; accepted July 26,1978 . 